Article

\title{
Suppression of Gene Juvenile Hormone Diol Kinase Delays Pupation in Heortia vitessoides Moore
}

\author{
Zihao Lyu, Zhixing Li, Jie Cheng, Chunyan Wang, Jingxiang Chen and Tong Lin *(1) \\ College of Forestry and Landscape Architecture, South China Agricultural University, Guangzhou 510642, China \\ * Correspondence: lintong@scau.edu.cn; Tel.: +86-020-8528-2217
}

Received: 5 July 2019; Accepted: 25 August 2019; Published: 2 September 2019

check for

updates

\begin{abstract}
Juvenile hormone diol kinase (JHDK) is a critical enzyme involved in juvenile hormone degradation in insects. In this study, HvJHDK in the Heortia vitessoides Moore (Lepidoptera: Crambidae) transcriptional library was cloned. Stage-specific expression patterns of HvJHDK, HvJHEH, and $\mathrm{HvJHE}$ as well as juvenile hormone titers were determined. The three tested enzymes participated in juvenile hormone degradation. Moreover, juvenile hormone titers peaked after larval-larval molts, consistent with a role for juvenile hormone in inhibition of metamorphosis. HvJHDK was subsequently suppressed using RNA interference (RNAi) to reveal its functions. Different concentrations of dsJHDK elicited the optimal interference efficiency at different life stages of $H$. vitessoides. Suppression of HvJHDK decreased HvJHDK content and increased the juvenile hormone titer, thereby resulting in reduced triglyceride content, sharply declined survival rate, clearly lethal phenotypes, and extended larval growth. Moreover, suppression of $H v J H D K$ upregulated $H v J H E H$ and $H v J H E$ expression levels, suggesting that there is feedback regulation in the juvenile hormone metabolic pathway. Taken together, our findings provide molecular references for the selection of novel insecticidal targets.
\end{abstract}

Keywords: juvenile hormone diol kinase; juvenile hormone; Heortia vitessoides Moore; RNA interference; triglyceride

\section{Introduction}

Juvenile hormone $(\mathrm{JH})$ is one of the most important insect hormones. This unique sesquiterpenoid hormone is crucial for various physiological processes in insects, including embryonic development, metamorphosis regulation, imaginal disc formation, sexual maturation, reproduction, pheromone production, and nutrition [1-5]. JH is mainly produced in the corpus allatum and released into the hemolymph, and through the hemolymph, $\mathrm{JH}$ is then transferred to target tissues via the carrier juvenile hormone-binding protein [6]. To date, seven natural JHs have been identified, and of those, JH III plays a primary physiological role [7,8]. In insects, JH synthesis and metabolism rates are tightly coupled with the JH titer balance $[9,10]$. Biosynthesis of $\mathrm{JH}$ involves the isoprenoid branch of the mevalonate (MVA) pathway. Biological functions of JH are realized when it binds to the heterodimeric receptor methoprene-tolerant (MET) and the nuclear receptor USP to induce protein kinase C signaling and calcium signal transduction [11]. At least three enzymes, namely, juvenile hormone esterase (JHE; EC 3.1.1.1) [12], juvenile hormone epoxide hydrolase (JHEH; EC 3.3.2.3) [13], and juvenile hormone diol kinase (JHDK; EC 2.1.7.3) [14], catalyze JH metabolism [15]. JHE converts JH to JH acid (JHa) via methyl ester hydrolysis, and JHEH hydrolyzes the JH epoxide moiety to produce JH diol (JHd). Alternatively, JHE catalyzes JHd, and JHEH degrades JHa to produce JH acid diol (JHad) [13]. In insects, cytoplasmic JHDK catalyzes JHd to JH diol phosphate (JHdp). Currently, JHad and JHdp are considered the principal final metabolites of JH degradation [14]. JHDK was first identified by Maxwell and colleagues, who isolated and purified Manse-JHDK from the Malpighian tubules of larval Manduca sexta. Manse-JHDK is active as a homodimer, is specific to JHd, prefers ATP over GTP, 
and requires $\mathrm{Mg}^{2+}$ for its activity; however, low concentrations of calcium ions inhibit Manse-JHDK activity [14]. Maxwell et al. performed cDNA cloning and confirmed that Manse-JHDK possessed elongation factor (EF)-hand motifs, as revealed by the amino acid sequence; moreover, they reported that three conserved sequence elements were involved in purine nucleotide binding, which were similar to GTP-binding proteins. To date, the majority of the studies on JH-metabolizing enzymes have focused on JHE and JHEH [16-21]. However, information regarding the roles of JHDK in the regulation of JH titers remains limited. In the present study, RNA interference (RNAi) of JHDK is reported for the first time in Heortia vitessoides Moore (Lepidoptera: Crambidae) to reveal the role of $J H D K$ in JH degradation in insects. The findings of this study can be useful in the selection of novel insecticidal targets.

H. vitessoides is a serious defoliator of the evergreen tree Aquilaria sinensis in tropical and subtropical regions. This tree has medicinal properties and provides raw material for the production of incense, which has enormous economic value. When $A$. sinensis is severely infested, $H$. vitessoides completely denudes the leaves [22], which leads to substantial financial loss. A comprehensive molecular understanding of H. vitessoides-a holometabolous insect-is thus warranted to help control this pest.

\section{Materials and Methods}

\subsection{Insects}

H. vitessoides was collected from Tianlu Lake Forest Park, Guangzhou and reared in a climatic cabinet at $26^{\circ} \mathrm{C}$ with $70-75 \%$ relative humidity and a $14 \mathrm{~L}: 10 \mathrm{D}$ photoperiod. H. vitessoides larvae were fed with $A$. sinensis leaves.

Mature larvae were transferred to a silt basin for harvesting pupae and adults. Newly emerged adults were fed with $7 \%$ honey solution. H. vitessoides eggs, 1-day-old first to 1-day-old third molting instars, 12-h-old fourth to 168-h-old fifth instars, 1- to 9-day-old pupae, and adults were collected to examine the stage-specific expression profiles of $H v J H D K, H v J H E H$, and $H v J H E$. The collected insects were immediately snap-frozen in liquid nitrogen and stored at $-80{ }^{\circ} \mathrm{C}$ until further use.

\subsection{RNA Extraction and $c D N A$ Synthesis}

Total RNA was extracted from each sample using the E.Z.N.A. ${ }^{\text {TM }}$ Total RNA Kit II (Omega Bio-Tek, Norcross, GA, USA), following the manufacturer's instructions. First-strand cDNA was synthesized from $2 \mu \mathrm{g}$ of total RNA from each sample using the PrimeScript ${ }^{\circledR}$ RT Reagent Kit with gDNA Eraser (Takara Bio, Otsu, Japan) and immediately stored at $-20^{\circ} \mathrm{C}$ until further use.

\subsection{Sequence Characterization and Phylogenetic Tree Analysis}

Based on the H. vitessoides transcriptome (SRX4045498), a putative unigene cDNA encoding JHDK was obtained. Open Reading Frame (ORF) Finder was used to acquire the cDNA sequence of HvJHDK ORF. Then, the corresponding pair of gene-specific primers (Table S1) was designed to amplify the HvJHDK ORF to verify the sequence. The GenBank accession numbers are listed in Table S2. PCR was conducted as follows: $5 \mathrm{~min}$ at $95^{\circ} \mathrm{C} ; 34$ cycles of $30 \mathrm{~s}$ at $95{ }^{\circ} \mathrm{C}, 30 \mathrm{~s}$ at $58^{\circ} \mathrm{C}$, and $2 \mathrm{~min}$ at $72{ }^{\circ} \mathrm{C}$; and $10 \mathrm{~min}$ at $72{ }^{\circ} \mathrm{C}$. Next, the PCR product was gel-purified, ligated into the pClone007 simple vector (TSINGKE Bio, Guangzhou, China), transformed into Escherichia coli DH5 $\alpha$ competent cells (Takara Bio, Otsu, Japan), and sequenced (TSINGKE Bio, Guangzhou, China). The identities of the recovered cDNAs of $H$. vitessoides were confirmed using BLASTx. JHDK amino acid sequences of other insect species were retrieved from the National Center for Biotechnology database. DNAMAN 6.0 (LynnonBiosoft, Quebec, QC, Canada) was used to edit sequences and perform multiple sequence alignment. Physicochemical properties of HvJHDK were predicted using ProtParam, and organization of domains in the sequences was predicted using SMART. A phylogenetic tree was constructed with MEGA- $X$ and ClustalX using the neighbor-joining method. 


\subsection{Synthesis of Double-Stranded (ds) RNA Targeting HvJHDK and Injection of dsRNA}

All reagents for dsRNA synthesis in the RNAi experiments were obtained from the T7 RiboMAX ${ }^{\mathrm{TM}}$ Express RNAi System Kit (Promega, Madison, WI, USA). Primers harboring T7 RNA polymerase promoter sequences were designed for PCR to obtain DNA templates. Primers used to synthetize dsRNA are listed in Table S1. DNA templates for HvJHDK and green fluorescent protein were used for a transcription reaction with T7 RNA polymerase to generate dsJHDK (258 bp) and dsGFP (400 bp) fragments. dsGFP was used as a negative control for nonspecific effects of dsRNA. The DNA template was removed, followed by dsRNA annealing and single-stranded RNA (ssRNA) removal through nuclease digestion. Next, the dsRNA was purified according to the manufacturer's protocol (Promega). After purification, the dsRNA was dissolved in nuclease-free water, quantified using a NanoDrop 2000 spectrophotometer (Thermo Fisher Scientific, Waltham, MA, USA), and confirmed using $1.5 \%$ agarose gel electrophoresis to ensure purity and integrity. All samples were stored at $-80{ }^{\circ} \mathrm{C}$ until further use.

To determine the optimal efficiency of dsJHDK RNAi, different concentrations of dsJHDK were injected into 12-h-old fourth (L4-injected group) and fifth instar (L5-injected group) larvae. The dsJHDK solution was diluted to $1,2,3,4$, and $5 \mu \mathrm{g} / \mu \mathrm{L}$ for injection. The volume injected per larva was $1 \mu \mathrm{L}$. Test insects were injected at the lateral internode membrane of the seventh and the eighth segments using FemtoJet (Eppendorf, Hamburg, Germany). The same concentration and volume of dsGFP and blanks were used as controls. A total of 30 larvae each were included in the treatment and the control groups to determine the interference efficiency of dsJHDK. A total of 40 larvae each were included in the L4-injected group, the L5-injected group, the dsGFP-injected group, and the CK (blank control, without any treatment) group to determine L4/L5 survival rate, pupation rate, and adult emergence rates, and experiments were repeated independently three times (consisting of 40 insects).

\subsection{Quantitative Real-Time PCR (RT-qPCR)}

Total RNA was extracted from each sample. First-strand cDNA was synthesized using the PrimeScript RT Reagent Kit with gDNA Eraser (Takara Inc., Dalian, China). The synthesized cDNA was then used as a template for RT-qPCR to verify the specificity and amplification efficiency of primers. Primer 5.0 was used for primer designing. Primers used for PCR are listed in Table S1. $\alpha$-tubulin [23] was used as an internal reference. The reaction system comprised $20 \mu \mathrm{L}$ cDNA template, $10 \mu \mathrm{L}$ TB

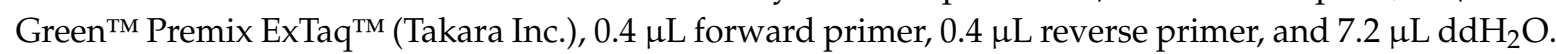
Non-template reactions (replacing cDNA with diethyl pyrocarbonate-treated water) were used as negative controls. Reaction conditions included initial denaturation at $95^{\circ} \mathrm{C}$ for $5 \mathrm{~min}$, followed by 40 cycles at $95^{\circ} \mathrm{C}$ for $10 \mathrm{~s}$ and $60^{\circ} \mathrm{C}$ for $20 \mathrm{~s}$. All the reactions were run using a LightCycler ${ }^{\circledR}$ Real-Time PCR System (Roche Diagnostics, Indianapolis, IN, USA). Relative expressions for each sample were calculated using the $2^{-\Delta \Delta C t}$ method [24]. Three biological replicates and three technical replicates were run for RT-qPCR analysis [25-30].

\subsection{Analysis of HvJHDK Protein Concentration}

After 36, 60, 84, and $108 \mathrm{~h}$ of dsRNA injection, the insects were prepared for protein content assays as follows. Surface dirt was removed with aseptic water, the insects were weighed, phosphate buffered saline (PBS) was added, and the insects were homogenized by hand. The samples were then centrifuged at 2000-3000 rpm for 20 min to separate the supernatant.

The Insect JHDK ELISA Kit (Shanghai Enzyme-linked Biotechnology Co., Ltd., Shanghai, China) was used to measure the JHDK protein concentrations. Purified insect JHDK antibody was used to coat microtiter plate wells and produce a solid-phase antibody. Then, samples were added to the wells, and the antibody was labeled with horseradish peroxidase (HRP) to form an antibody-antigen-enzyme-antibody complex. After thorough washing, 3,3' 5, 5' -tetramethylbenzidine (TMB) substrate solution was added; TMB substrate turns blue when catalyzed by HRP. The reaction 
was terminated by adding sulfuric acid, and the color change was measured at $450 \mathrm{~nm}$ using the Varioskan LUX Multimode Microplate Reader (Thermo Fisher Scientific, Waltham, MA, USA).

The total JHDK concentration in the samples was determined by extrapolating the optimal density of the samples on the linear regression equation of the standard curve.

\subsection{Analysis of Triglyceride (TG) Content}

The lipid of the insect is mainly in the form of triglyceride (TG). To identify the TG content, living 5-day-old fifth instar larvae and 1-day-old pupae injected with dsJHDK were collected and homogenized. The homogenates $(10 \% \mathrm{w} / \mathrm{v})$ were centrifuged at $2500 \mathrm{rpm}\left(4^{\circ} \mathrm{C}\right)$ for $10 \mathrm{~min}$, and the supernatant was stored at $4{ }^{\circ} \mathrm{C}$ until analysis. PBS $(0.1 \mathrm{~mol} / \mathrm{L}, \mathrm{pH} 7.4)$ was used as the homogenate medium. TG content was determined using a triglyceride assay kit (Nanjing Jiancheng Bioengineering Institute, Nanjing, China) following the manufacturer's instructions. Sample optical density (OD) values were determined by measuring absorbance at $510 \mathrm{~nm}$ using the Varioskan ${ }^{\mathrm{TM}}$ LUX multimode microplate reader (Thermo Fisher Scientific). The TG content was normalized with the total protein content, which was determined using the Bradford Protein Assay Kit (Nanjing Jiancheng Bioengineering Institute, Nanjing, China). Three independent replicates were used for the assays.

\subsection{Measurement of JH Titer}

The $\mathrm{JH}$ titer of $H$. vitessoides was determined using reversed-phase high-performance liquid chromatography (RP-HPLC) following the method reported by Zhang [20]. After accurately weighing them, the insects were homogenized in methanol:diethyl ether at an equal volume ratio, followed by ultrasonic crushing of the homogenate for $10 \mathrm{~min}$ to completely extract JH. Next, $2 \mathrm{~mL}$-hexane was added, followed by ultrasonic oscillation at $4{ }^{\circ} \mathrm{C}$. The supernatant was centrifuged at $12,000 \mathrm{rpm}$ for $10 \mathrm{~min}$. Extraction was repeated thrice, and the supernatants were combined. The resulting supernatant was dried by blowing under high purity nitrogen. After drying, the supernatant was dissolved in $500 \mu \mathrm{L}$ chromatography grade methanol, filtered through a $0.22 \mu \mathrm{m}$ organic membrane, sealed in a $2 \mathrm{~mL}$ sampling bottle, and stored at $-20^{\circ} \mathrm{C}$ until use. The mobile phase used was $80 \%$ methanol (methanol:water $=80: 20$ ) at a mobile phase flow rate of $0.8 \mathrm{~mL} / \mathrm{min}$, UV detector wavelength $(\lambda)$ of $218 \mathrm{~nm}$, and sample volume of $20 \mu \mathrm{L}$. LC20AD (Shimadzu, Shanghai, China) Zorbax Extend-C18 (Agilent, Beijing, China) reversed-phase column $(4.6 \times 250 \mathrm{~mm})$ was used. The JH III standard was purchased from Sigma. The JH titers in each sample were calculated by extrapolating the values on the linear regression equation of the standard curve. Three independent biological replicates were used for the assays.

\subsection{Phenotype Analysis}

The injected individuals were checked frequently and carefully for possible phenotypic changes. The insects that did not react to brush touching within a minute were regarded as dead. In this species, the initiation of pupation is indicated by formation of a pupal chamber. To check for pupation, a small slit was made in the pupal chamber with a blade. The living individuals were continuously kept in soil to check for adult emergence. The emergence rates were evaluated during a 3 week observation period.

\subsection{Statistical Analysis}

Gene expression data are presented as the means \pm standard deviation (SD) for three independent replicates. Statistical package for the social sciences v18 (SPSS; IBM, NewYork, NY, USA) was used for one-way analysis of variance and Duncan's multiple comparisons. A Student's t-test was performed to compare the different expressions between the controls and the treated individuals. GraphPad Prism 5 was used to create figures. 


\section{Results}

\subsection{HvJHDK Characterization and Phylogenetic Analysis}

Based on the $H$. vitessoides transcriptome, the putative cDNA sequence of JHDK was obtained and designated as HvJHDK (GenBank: MK561746). This sequence was 531 bp long and encoded a 176 amino acid protein with a theoretical molecular weight of $19.83 \mathrm{kDa}$ and an isoelectric point of 4.60. The instability index of the protein was 22.4, indicating that it was stable. Three predicted GTP-binding sites and three predicted calcium-binding motifs, which are highly conserved among JHDK proteins, were present in the deduced protein sequence (Figure 1) [8,31]. Sequence alignment using DNAMAN revealed that $H v J H D K$ had a high sequence similarity with other insect JHDKs. A phylogenetic tree was constructed to display the relationship among insect JHDKs (Figure 2). Both HvJHEH and HvJHE were identified in the same way.

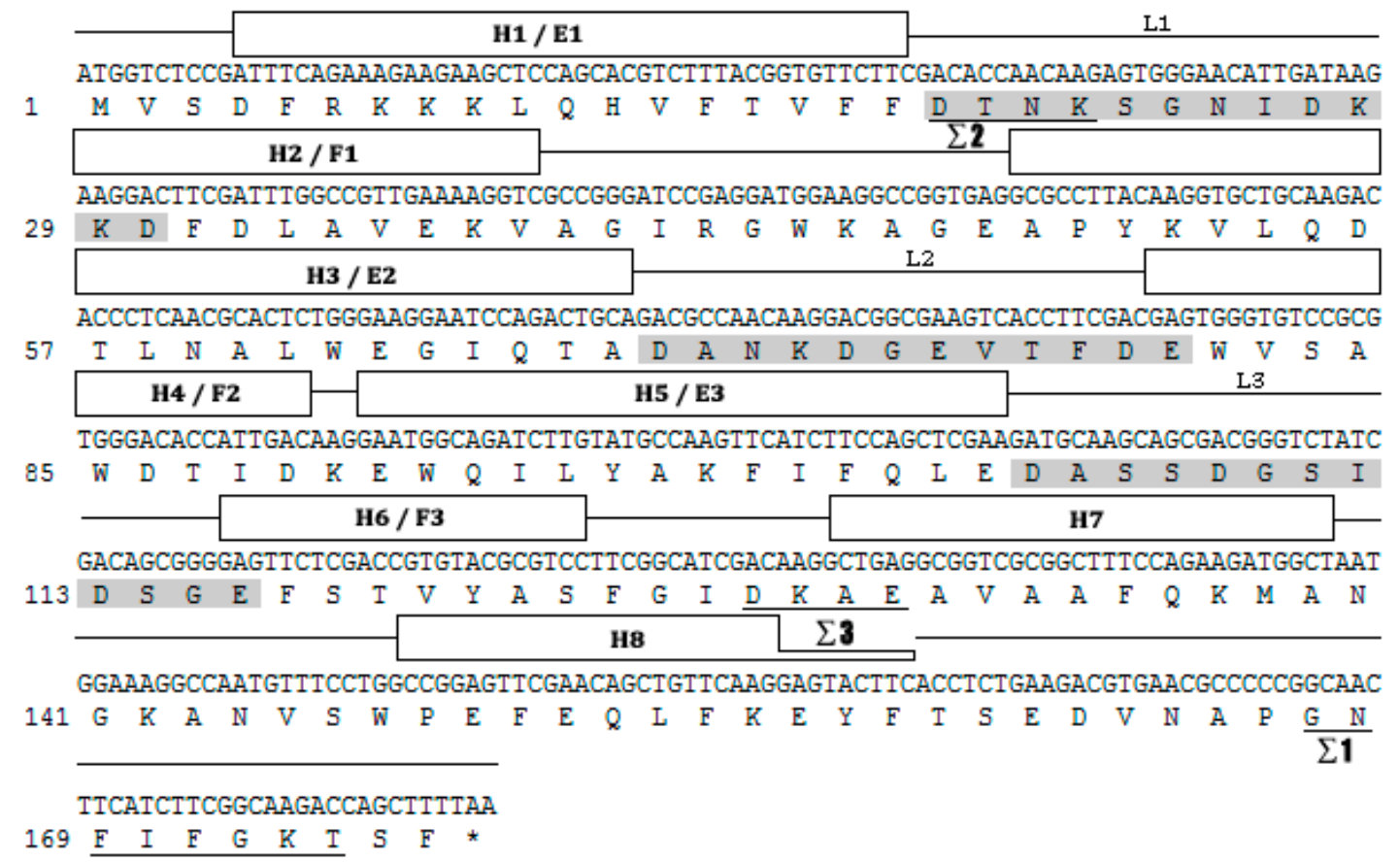

Figure 1. Nucleotide and deduced amino acid sequences of juvenile hormone diol kinase Heortiavitessoides (HvJHDK). The three potential fingerprint motifs (i.e., $\Sigma 1-3$ ) of GTP-binding proteins are underlined, and the three elongation factor (EF)-hand motifs (calcium-binding motifs) are presented in shaded boxes. The $\alpha$-helical sections (H1-8) are boxed and connected with lines, indicating loops or unstructured regions. E1-L1-F1, E2-L2-F2, and E3-L3-F3 form three putative EF hands, i.e., helix-loop-helix structural domains.

\subsection{RNAi Efficiency for HvJHDK}

As expected, compared with control expression, $H v J H D K$ expression was knocked down after injection. A concentration of $3 \mu \mathrm{g} / \mu \mathrm{L}$ dsJHDK showed the highest RNAi efficiency $36 \mathrm{~h}$ after injection in the L4-injected group, with an optimal interference of approximately $60 \%$ (Figure 3A). A concentration of $4 \mu \mathrm{g} / \mu \mathrm{L}$ dsJHDK showed the highest RNAi efficiency $60 \mathrm{~h}$ after injection in the L5-injected group, with an optimal interference of approximately $73 \%$ (Figure 3B). Based on these findings, we continued injecting concentrations of 3 and $4 \mu \mathrm{g} / \mu \mathrm{L}$ into 12-h-old L4 and L5 larvae, respectively, as follow-up experiments. 


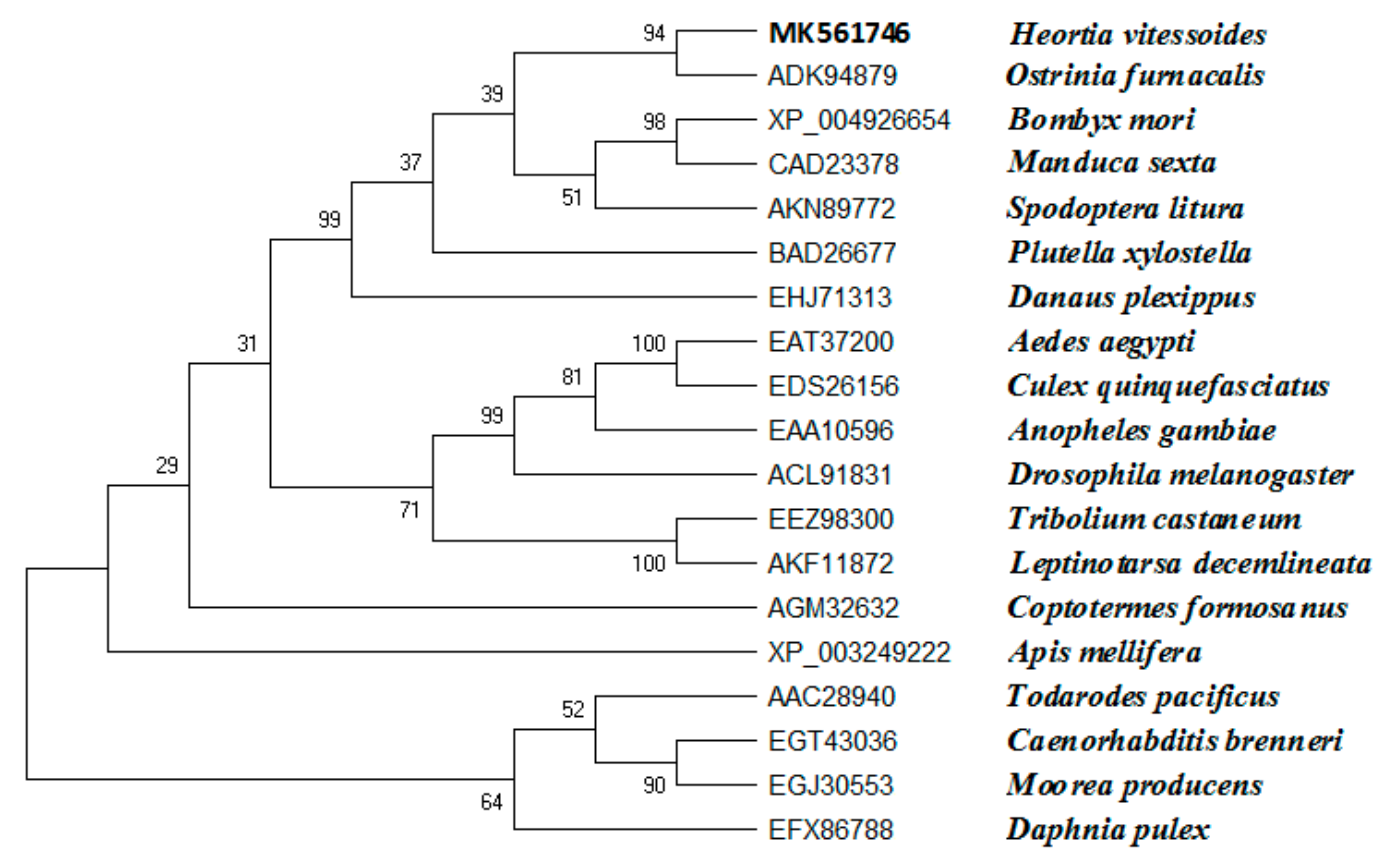

Figure 2. Phylogenetic analysis of juvenile hormone diol kinase homologs from 19 insect species based on amino acid sequences. A phylogenic tree was constructed using MEGA-X with sequences obtained from GenBank. Bootstrap analysis results of 1000 replicates are shown.


Figure 3. Assessment of the efficiency of RNA interference. (A) Relative expression levels of HvJHDK in fourth instar larvae after injection of doses of $1,2,3,4$, and $5 \mu \mathrm{g} / \mu \mathrm{L}$. (B) Relative expression levels of $H v J H D K$ in fifth instar larvae after injection of doses of $1,2,3,4$, and $5 \mu \mathrm{g} / \mu \mathrm{L}$. Different letters indicate significant differences at $p<0.05$ according to one-way analysis of variance (ANOVA). The data represent the mean $\pm \operatorname{SD}(n=3)$.

After 36, 60, 84, and $108 \mathrm{~h}$ of injection in the L5-injected group, we assessed the HvJHDK concentration in the whole body of the insects. The HvJHDK concentration was significantly reduced in all cases except after $36 \mathrm{~h}$ of injection (Figure 4 ). These results substantiated the success of RNAi by $\mathrm{ds} J H D K$ injection in terms of protein translation. 


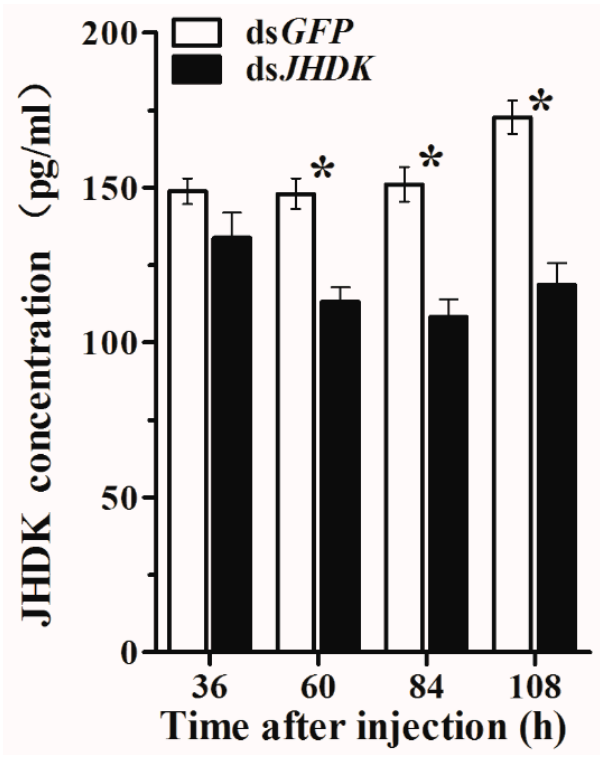

Figure 4. Changes in HvJHDK concentration after injection in L5-injected individuals. The data represent the mean $\pm \operatorname{SD}(n=3)$. The asterisk $\left({ }^{*}\right)$ indicates a significant difference according to Student's t-test $(p<0.05)$.

\subsection{JH titers and Developmental Stage-Specific Expression of $\mathrm{HvJHDK}, \mathrm{HvJHEH}$, and $\mathrm{HvJHE}$}

In this study, we determined the JH titers at different development stages of H. vitessoides (Figure 5). From the first to the fourth instar stages, the JH titers peaked immediately after molting. The highest peak at L4D0 reached a value of $208.5 \mathrm{ng} / \mathrm{mg}$. In contrast, the JH titer was the lowest at the final stage of molting, which is consistent with its function of inhibiting metamorphosis. The JH titer was upregulated after the suppression of its degradation gene $H v J H D K$.

$H v J H D K, H v J H E$, and $H v J H E H$ were consistently expressed at all tested developmental stages (Figure 5). The highest peak of HvJHDK expression was detected in 72-h-old fifth instar larvae. However, at all stages, the expression of $H v J H E$ was relatively lower than those of $H v J H E H$ and $H v J H D K$. The expression of $H v J H E H$ was lower than that of $H v J H D K$ at all stages. Following suppression of $H v J H D K$, both $H v J H E$ and $H v J H E H$ were upregulated.

\subsection{Phenotype Analysis after RNAi}

Representative phenotypes of the larvae after dsJHDK and dsGFP injections were examined (Figure 6). Obvious abnormal/lethal phenotypes, i.e., darkened body color and insects trapped in the old cuticle during molting, were observed. The total developmental duration of L5-injected insects from the fifth instar stage to pupation increased from 7.5 to 8.5 days. The rate of growth is presented in Figure 7. Pupal weight slightly increased in the treatment group compared to the control groups (Table 1).

Table 1. Effect of dsJHDK injection on the growth status of Heortia vitessoides. CK, controls; L4-injected, dsJHDK injected to 12-h-old fourth instar larvae; L5-injected, dsJHDK injected to 12-h-old fifth instar larvae. The pupal weight indicated was measured at 1 day of age. Data are presented as mean \pm $\mathrm{SD}(\mathrm{n}=3)$. The different letters indicate significant differences among the treatments and the control measured at the same time $(p<0.05)$.

\begin{tabular}{ccccc}
\hline & CK & dsGFP & L4-Injected & L5-Injected \\
\hline Pupal weight $(\mathrm{mg})$ & $124.5 \pm 2.9 \mathrm{ab}$ & $119.0 \pm 1.9 \mathrm{~b}$ & $130.6 \pm 1.9 \mathrm{a}$ & $128.6 \pm 2.6 \mathrm{a}$ \\
L4-L5 survival rate (\%) & $100 \pm 0.00 \mathrm{a}$ & $98.3 \pm 2.89 \mathrm{a}$ & $83.3 \pm 5.77 \mathrm{~b}$ & \\
Pupation rate (\%) & $96.7 \pm 2.89 \mathrm{a}$ & $93.2 \pm 3.20 \mathrm{ab}$ & $82.2 \pm 16.08 \mathrm{ab}$ & $75.0 \pm 13.29 \mathrm{~b}$ \\
Adult emergence rate (\%) & $95.0 \pm 5.00 \mathrm{a}$ & $92.8 \pm 2.88 \mathrm{a}$ & $53.6 \pm 3.23 \mathrm{~b}$ & $38.1 \pm 3.24 \mathrm{c}$ \\
\hline
\end{tabular}



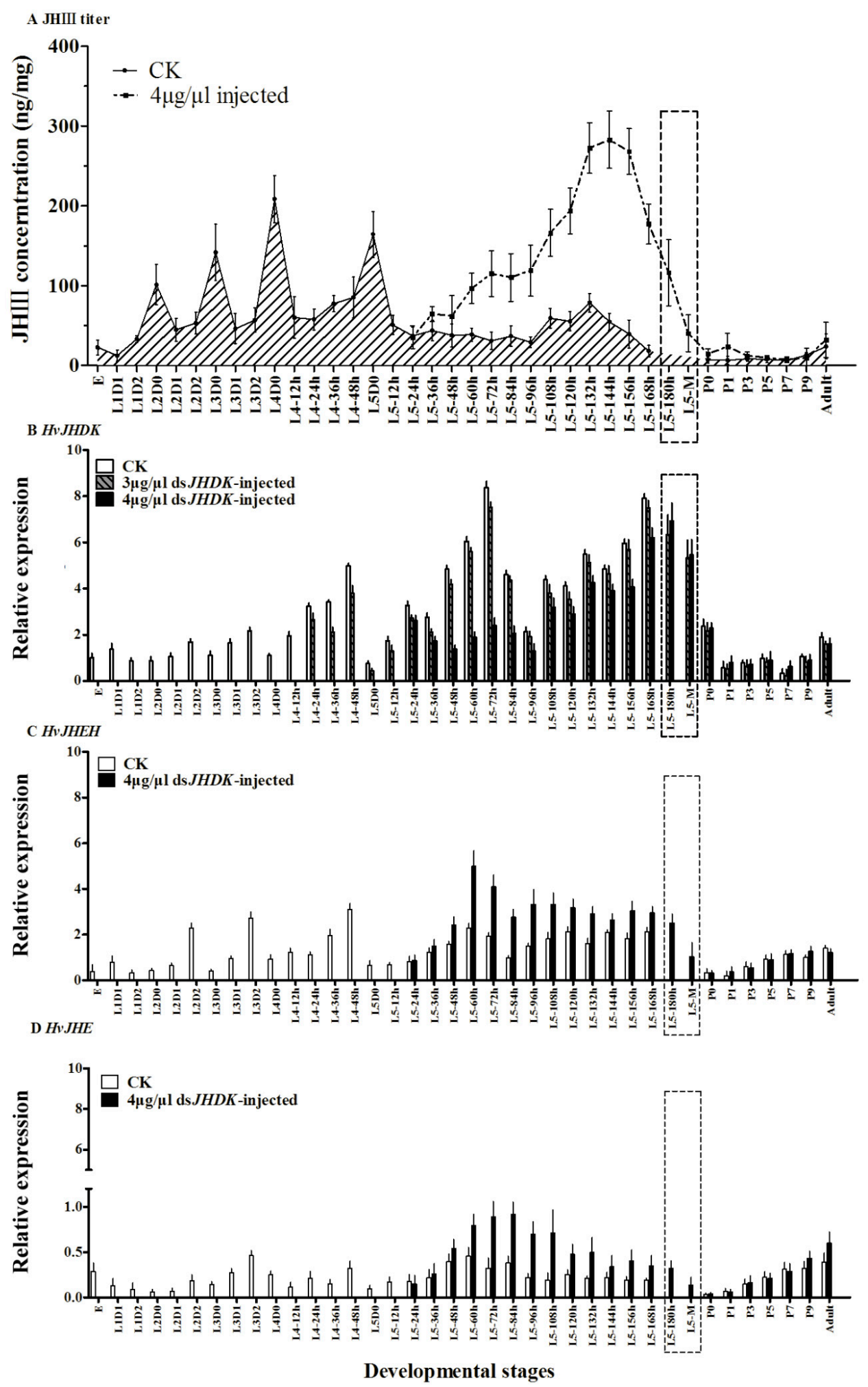

Figure 5. Juvenlie hormone (JH) titer (A) and expression profiles of $H v J H D K(\mathbf{B})$, juvenile hormone epoxide hydrolase $(J H E H)(\mathbf{C})$, and juvenile hormone esterase (JHE) (D) at different development stages. E, eggs; L1D1-L4D0, 1-day-old first-fourth instar larvae immediately after molting; L4-12h-L5-168h, 12-h-old fourth to 168-h-old fifth instar larvae; P0-P9, pupae immediately after molting to 9-day-old pupae; A, adults. Extended final larval stages are marked using square frames. CK, blank control. The data represent the mean $\pm S D(n=3)$. 


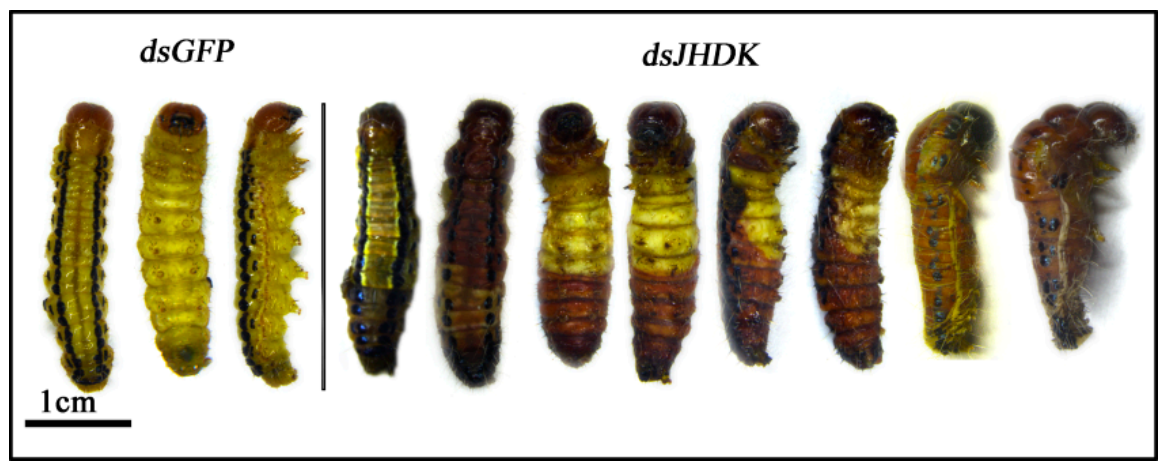

Figure 6. Lethal phenotypes caused by RNA interference for HvJHDK.

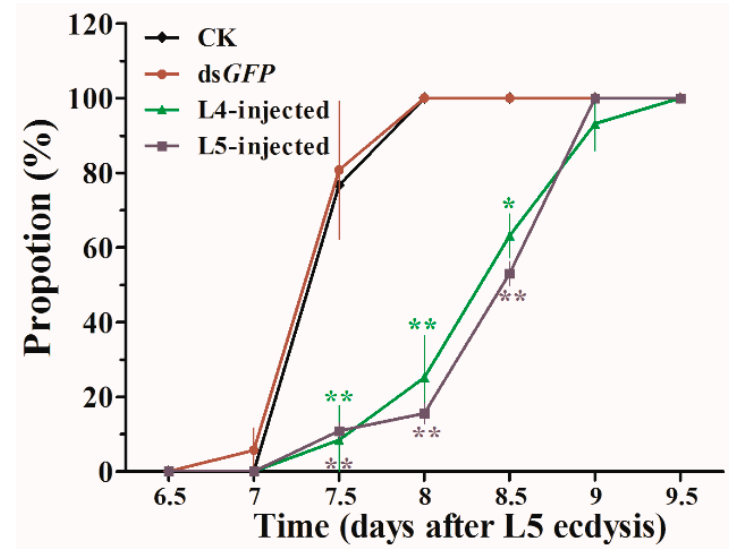

Figure 7. Loss of HvJHDK function resulted in extended larval periods. Proportion refers to the percentages of larvae in the different treatment groups that ecdyzed to the pupal stage. Dead larvae were not included in the statistical analyses. Data are presented as mean $\pm \operatorname{SD}(n=3)$. The asterisk indicates a significant difference according to Student's t-test $\left({ }^{*} p<0.05 ;{ }^{* *} p<0.01\right)$.

\subsection{Relative Gene Expression after RNAi}

We tested the relative gene expression after dsJHDK injection at $72 \mathrm{~h}$ via RT-qPCR. As shown in Figure 8, the expressions of HvJHEH, HvJHE, Krüppel homolog 1 (Hvkr-h1), and HvMET were increased, while there was no significant change in the expression level of JH acid methyltransferase (HvJHAMT).

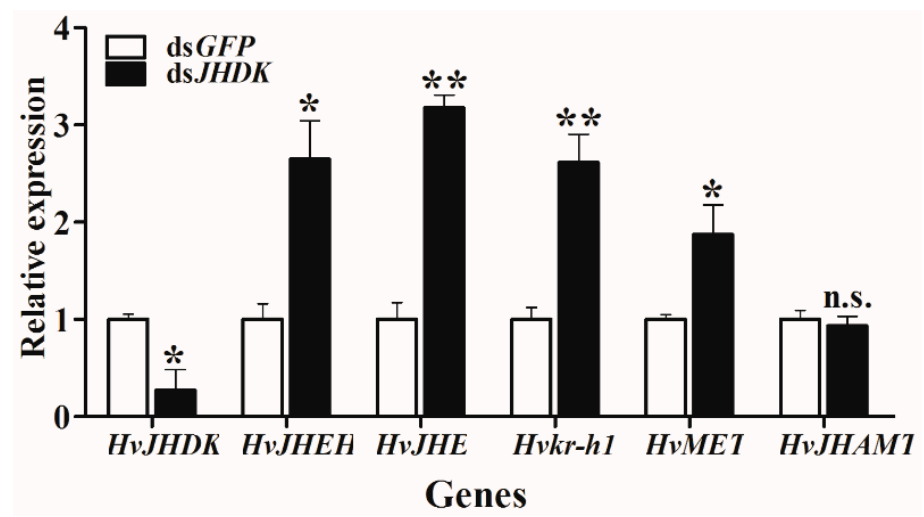

Figure 8. Relative gene expression in $d s J H D K$-injected larvae versus dsGFP-injected larvae. Expression levels of the JH metabolic and response genes HvJHDK, HvJHEH, HvJHE, JH acid methyltransferase (HvJHAMT), HvMET, and Krüppel homolog 1 (Hvkr-h1) were investigated $72 \mathrm{~h}$ after injection of dsRNA. The data represent the mean $\pm \mathrm{SD}(\mathrm{n}=3)$. The asterisk indicates a significant difference according to Student's t-test $\left({ }^{*} p<0.05 ; * * p<0.01 ;\right.$ n.s. not significant). 


\title{
3.6. Analysis of TG Content and the Expression of Lipid Accumulation and Degradation-Related Genes
}

We tested the TG content after dsJHDK injection at $108 \mathrm{~h}$ and $204 \mathrm{~h}$, respectively, to identify the changes in TG content induced by suppression of HvJHDK. The results showed that the TG content of the insect decreased at $108 \mathrm{~h}$ and $204 \mathrm{~h}$ after injection of dsJHDK. We also tested the expression of lipid accumulation and degradation-related genes after dsJHDK injection at $72 \mathrm{~h}$ via RT-qPCR. The results showed that the expression of alcohol dehydrogenase ( $H v A D H)$ gene was decreased, and the expression of adipose triglyceride lipase (HvATGL), triglyceride lipase (HvTGL), and lipase 1 (HvLIP1) genes were increased (Figure 9).
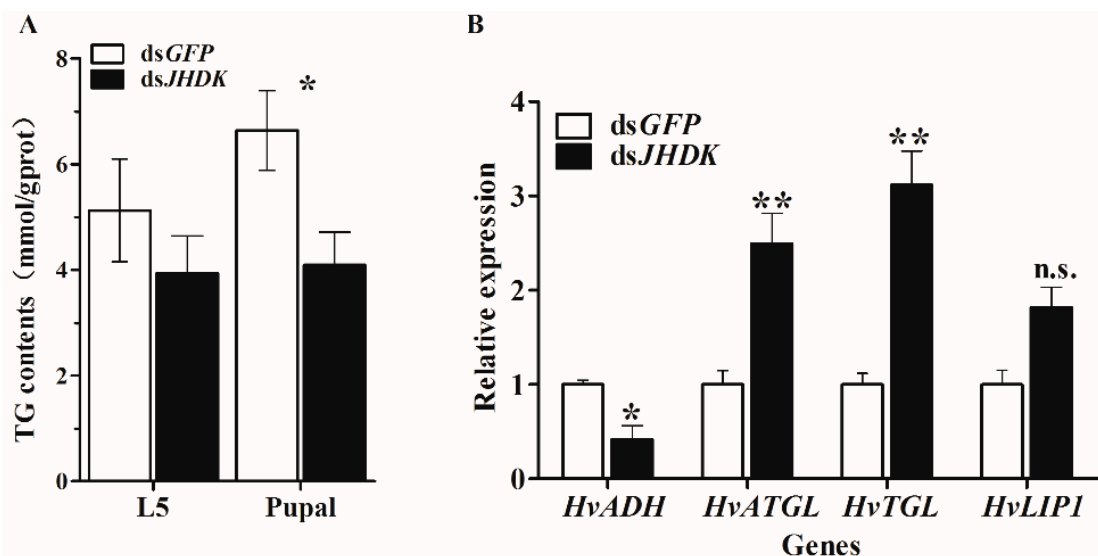

\begin{abstract}
Figure 9. Analysis of triglyceride (TG) content. (A) Assessment of TG content in fifth instar larvae and pupae after injection at $108 \mathrm{~h}$ and $204 \mathrm{~h}$, respectively. The data represent the mean \pm SD $(n=3)$, each repetition contained 10 individuals. (B) Relative gene expression of the lipid accumulation and the degradation-related genes alcohol dehydrogenase $(H v A D H)$, adipose triglyceride lipase (HvATGL), triglyceride lipase (HvTGL), and lipase 1 (HvLIP1) $72 \mathrm{~h}$ after injection of dsRNA. The data represent the mean $\pm \mathrm{SD}$ $(\mathrm{n}=3)$. The asterisk indicates a significant difference according to Student's t-test $\left({ }^{*} p<0.05 ;{ }^{* *} p<0.01\right.$; n.s. not significant).
\end{abstract}

\section{Discussion}

JHDK is an important enzyme that metabolizes JH in insects. In this study, a novel cDNA sequence of JHDK was designated from the H. vitessoides transcriptional library. Homologous alignment and phylogenetic tree analyses revealed that HvJHDK is highly homologous with JHDKs in other insect species. The well conserved GTP-binding protein motifs and calcium-binding sites of JHDKs [6,31] were present in the predicted protein sequence of HvJHDK. These results suggest that HvJHDK is highly similar to JHDKs in other insects.

To explore the functions of JHDK using RNAi, we first determined the optimal interference concentration. It was necessary to determine the optimal time point and concentration for application for the following RNAi experiments. We found that the sensitivity of the fourth and the fifth instar larvae to dsJHDK differed, and that injections of 3 and $4 \mu \mathrm{g} / \mu \mathrm{L}$ of dsJHDK produced the optimal interference efficiency in fourth and fifth instar larvae, respectively. The interference effect was found to last for approximately $180 \mathrm{~h}$ (including extended final larval stage) in the L5-injected larvae $(4 \mu \mathrm{g} / \mu \mathrm{L})$. The interference efficiency of dsJHDK $(<10 \%)$ recovered faster in the L4-injected larvae $(3 \mu \mathrm{g} / \mu \mathrm{L})$, with the effects lasting for approximately $108 \mathrm{~h}$. (Figure $5 \mathrm{~B})$. The expression levels of the introduced corresponding genes of dsRNA are generally believed to return to the original level after being degraded. Furthermore, both L4- and L5-injected larvae showed lower adult emergence rates than pupation and L4-L5 survival rates. In H. vitessoides, the adult emergence process might be more dependent on HvJHDK for it to exert its effects. Abnormal elevation of the JH titer inhibiting the growth of imaginal discs may be one reason underlying the low adult emergence rates [2]. 
$\mathrm{JH}$ plays an important role in regulating growth and development in larval insects, and $\mathrm{JH}$ degradation is specific to developmental stages, coinciding with its functions at each stage [32]. In the present study, we systematically assessed the expression patterns of the three JH degradation genes $H v J H D K, H v J H E H$, and $H v J H E$ and measured the JH titers at different developmental stages. These developmental profiles are invaluable because the timing of changes in the JH titers (HvJHDK induction) may correlate with changes in the status of physiological events [33]. The JH titers in $H$. vitessoides, which vary with developmental stages, peaked immediately after molting (L2D0, L3D0, L4D0, and L5D0), and JH secretion gradually decreased at the end of the fifth instar stage, consistent with its role in metamorphosis inhibition. Similar to results obtained in Helicoverpa armigea [20], the expression of HvJHDK (L3D0, L4D0, and L5D0), HvJHEH (L3D0, L4D0, and L5D0), and HvJHE (L2D0, L3D0, L4D0, and L5D0) decreased after larval molting and increased during larval feeding. The expression time frames of $\mathrm{HvJHDK}, \mathrm{HvJHEH}$, and $\mathrm{HvJHE}$ are analogous. The negative correlation between the expression of $\mathrm{JH}$ degradation enzymes and the $\mathrm{JH}$ titers suggests that these three genes play roles in insect molting by regulating the JH titers. In addition, all three JH degradation genes were highly expressed during the L5 instar stage. Based on these findings, we expect that maintaining high expression levels of these three genes is vital for successful larval-pupal metamorphosis. In holometabolous insects, a pulse of $20 \mathrm{E}$ and high $\mathrm{JH}$ titer elicited larval-larval molting, whereas a pulse of $20 \mathrm{E}$ and low JH titer during the final larval instar stage triggered larval-pupal metamorphosis [32]. Furthermore, in the present study, the expression of $H v J H D K, H v J H E$, and $H v J H E H$ and the titers of $\mathrm{JH}$ were higher in adults than in pupae. During larval molting, $\mathrm{JH}$ prevents metamorphosis and plays roles in the formation and the development of yolk in adult females. It also plays roles in the development of accessory glands and mating ability in adult males [34].

Suppression of HvJHDK increased the JH titers, maintained the increased expression of two JH degradation enzymes (Figure 5), and increased the expression of the two JH response genes HvMET and Hvkr-h1. The expression of HvJHAMT (the last key enzyme in JH synthesis) did not show any significant change (Figure 8). In Bombyx mori JHE knockouts, the expression of JHEH and JHDK increased by 1.8 and 4.1 fold, respectively [19]; in Nilaparvata lugens, Nljhdk knockdown upregulated Nljhe and Nljheh expression [35], whereas Nljheh knockdown upregulated Nljhe expression [21]. Taken together, these findings indicate that there exists a complementary approach in the JH metabolic pathway such that inactivation of one enzyme leads to activation of another. Furthermore, suppression of HvJHDK extends the larval duration, and this result is similar to those of LdJHDK, LdJHEH1, LdJHEH2 [8,16], and JHE knockdown in Leptinotarsa decemlineata and B. mori [19], which also mediated an abnormal rise in the JH titer in vivo. However, suppression of HvJHDK induced an increase in the pupal weights, similar to the effect of JHE knockdown but contrary to that of LdJHDK knockdown [8]. Abnormal rise in the JH titers may lead to different results between Lepidoptera and Coleoptera. JH may regulate the body size of $H$. vitessoides by mediating the growth duration, as observed in M. sexta [36].

During the final larval instar stage, the commitment to undergo larval-pupal molting requires the action of ecdysteroids in the absence of JH [37]. After suppression of HvJHDK, which induced an abnormal increase of the JH titer, we observed some lethal phenotypes and reduced survival rate in $\mathrm{H}$. vitessoides. Previous studies revealed that $\mathrm{JH}$ treatment alone suppresses the expression of an epidermal protein associated with cuticle formation [33]. JH also regulates polyphenisms, such as color morphs and caste differentiation [38]. In organisms, enzymes integrate complex metabolic reactions and form an orderly network; changes in the key enzymes directly affect the metabolism of individuals and may even lead to death. Of course, physiological defects in affected insects warrant further research.

Because suppression of $H v J H D K$ leads to delayed growth and development, we speculate that HvJHDK mediates nutrition and energy metabolism by regulating the JH titers. In this study, TG content decreased in the larval and the pupal stages after 108 and $204 \mathrm{~h}$ of injection, respectively (Figure 9). Relative expressions of the lipid accumulation-related gene $H v A D H$ were decreased, whereas those of the lipid metabolism pathway genes HvATGL and HvTGL and the lipid degradation-related gene 
HvLIP1 were increased. Lipids provide energy for growth during long non-feeding periods and are extremely important for the growth and the reproduction of insects [39]. Our findings suggest that the $\mathrm{JH}$ degradation pathway mediated by HvJHDK promotes lipid accumulation in both the larval and the pupal stages of $H$. vitessoides, and suppression of $H v J H D K$ restrains accumulation of TG content. A decrease in TG content resulting in insufficient energy supply during the pupal period could be one of the reasons underlying the low adult emergence rate $(53.6 \%$ and $38.1 \%$ of L4-injected and L5-injected insects, respectively). Furthermore, in Cnaphalocrocis medinalis, the TG content was lower in treated adult females than in control adult females $2-4$ days after the treatment with $\mathrm{JH}$ analog [40]. However, the other JHDK-mediated pathways regulating insect growth need to be further studied.

\section{Conclusions}

In summary, we obtained and identified the HvJHDK sequence from the $H$. vitessoides larval transcriptional library. JH titers at a spectrum of developmental stages, expression patterns of $H v J H D K$, $H v J H E H$, and $H v J H E$, and expression of $H v J H D K, H v J H E H$, and $H v J H E$ after suppression of $H v J H D K$ were assessed. Feedback regulation may exist in the JH metabolic pathway. Moreover, 3 and $4 \mu \mathrm{g} / \mu \mathrm{L}$ were identified as the optimal concentrations for inducing the maximum RNAi efficiency in L4-and L5-injected insects, respectively. Suppression of HvJHDK reduced the HvJHDK protein concentration, increasing the JH titer and decreasing the TG content; this may explain the development of extended larval growth, lethal phenotype, and reduced pupation and adult emergence rates. These findings provide a basis for further studying the functions of HvJHDK in the growth and the metabolism of H. vitessoides.

Supplementary Materials: The following are available online at http://www.mdpi.com/2075-4450/10/9/278/s1, Table S1: PCR primers used in this study; Table S2: GenBank accession number in this study.

Author Contributions: Data curation, Z.L. (Zihao Lyu); Funding acquisition, T.L.; Investigation, Z.L. (Zihao Lyu), Z.L. (Zhixing Li) and J.C. (Jie Cheng); Methodology, Z.L. (Zihao Lyu) and J.C. (Jingxiang Chen); Software, Z.L. (Zihao Lyu), J.C. (Jie Cheng) and C.W.; Visualization, Z.L. (Zhixing Li) and C.W.; Writing—original draft, Z.L. (Zihao Lyu); Writing—review \& editing, T.L.

Funding: This work was supported by grants from the National Natural Science Foundation of China (31470653) and the Natural Science Foundation of Guangdong Province, China (2015A030313416).

Conflicts of Interest: The authors declare no conflict of interest.

\section{References}

1. Riddiford, L.M.; Truman, J.W.; Mirth, C.K.; Shen, Y.C. A role for juvenile hormone in the prepupal development of Drosophila melanogaster. Development 2010, 137, 1117-1126. [CrossRef]

2. Truman, J.W.; Hiruma, K.; Allee, J.P.; MacWhinnie, S.G.B.; Champlin, D.T.; Riddiford, L.M. Juvenile hormone is required to couple imaginal disk formation with nutrition in Insects. Science 2006, 312, 1385-1388. [CrossRef]

3. Wyatt, G.R.; Davey, K.G. Cellular and Molecular Actions of Juvenile Hormone. II. Roles of Juvenile Hormone in Adult Insects. Adv. Insect Physiol. 1996, 26, 1-155.

4. Bellés, X.; Martín, D.; Piulachs, M. The mevalonate pathway and the synthesis of juvenile hormone in insects. Anпu. Rev. Entomol. 2005, 50, 181-199. [CrossRef]

5. Li, Y.; Hernandez-Martinez, S.; Unnithan, G.C.; Feyereisen, R.; Noriega, F.G. Activity of the corpora allata of adult female Aedes aegypti: Effects of mating and feeding. Insect Biochem. Mol. Biol. 2003, 33, 1307-1315. [CrossRef]

6. Prestwich, G.D.; Wojtasek, H.; Lentz, A.J.; Rabinovich, J.M. Biochemistry of proteins that bind and metabolize juvenile hormones. Arch. Insect Biochem. Physiol. 1996, 32, 407-419. [CrossRef]

7. Steiner, B.; Pfister-Wilhelm, R.; Grossniklaus-Bürgin, C.; Rembold, H.; Treiblmayr, K.; Lanzrein, B. Titres of juvenile hormone I, II and III in Spodoptera littoralis (Noctuidae) from the egg to the pupal molt and their modification by the egg-larval parasitoid Chelonus inanitus (Braconidae). J. Insect Physiol. 1999, 45, 401-413. [CrossRef] 
8. Fu, K.Y.; Lü, F.G.; Guo, W.C.; Li, G.Q. Characterization and functional study of a putative juvenile hormone diol kinase in the Colorado potato beetle Leptinotarsa decemlineata (Say). Arch. Insect Biochem. Physiol. 2015, 90, 154-167. [CrossRef]

9. Li, S.; Falabella, P.; Kuriachan, I.; Vinson, S.B.; Borst, D.W.; Malva, C.; Pennacchio, F. Juvenile hormone synthesis, metabolism, and resulting hemolymph titer in Heliothis virescens larvae parasitized by Toxoneuron nigriceps. J. Insect Physiol. 2003, 49, 1021-1030. [CrossRef]

10. Gilbert, L.I.; Granger, N.A.; Roe, R.M. The juvenile hormones: Historical facts and speculations on future research directions. Insect Biochem. Mol. Biol. 2000, 30, 617-644. [CrossRef]

11. Davey, K. From insect ovaries to sheep red blood cells: A tale of two hormones. J. Insect Physiol. 2007, 53, 1-10. [CrossRef]

12. Hammock, B.D.; Sparks, T.C. A rapid assay for insect juvenile hormone esterase activity. Anal. Biochem. 1977, 82, 573-579. [CrossRef]

13. Share, M.R.; Roe, R.M. A partition assay for the simultaneous determination of insect juvenile hormone esterase and epoxide hydrolase activity. Anal. Biochem. 1988, 169, 81-88. [CrossRef]

14. Maxwell, R.A.; Welch, W.H.; Horodyski, F.M.; Schegg, K.M.; Schooley, D.A. Juvenile hormone diol kinase II. Sequencing, cloning, and molecular modeling of juvenile hormone-selective diol kinase from Manduca sexta. J. Biol. Chem. 2002, 277, 21882-21890. [CrossRef]

15. Li, S.; Jiang, R.; Cao, M. Metabolism of juvenile hormone. Acta Entomol. Sin. 2004, 47, 389-393.

16. Lü, F.; Fu, K.Y.; Guo, W.C.; Li, G.Q. Characterization of two juvenile hormone epoxide hydrolases by RNA interference in the Colorado potato beetle. Gene 2015, 570, 264-271. [CrossRef]

17. Tsubota, T.; Minakuchi, C.; Nakakura, T.; Shinoda, T.; Shiotsuki, T. Molecular characterization of a gene encoding juvenile hormone esterase in the red flour beetle, Tribolium castaneum. Insect Mol. Biol. 2010, 19, 527-535. [CrossRef]

18. Zhang, Q.R.; Xu, W.H.; Chen, F.S.; Li, S. Molecular and biochemical characterization of juvenile hormone epoxide hydrolase from the silkworm, Bombyx mori. Insect Biochem. Mol. Biol. 2005, 35, 153-164. [CrossRef]

19. Zhang, Z.; Liu, X.J.; Shiotsuki, T.; Wang, Z.S.; Xu, X.; Huang, Y.P.; Li, M.W.; Li, K.; Tan, A.J. Depletion of juvenile hormone esterase extends larval growth in Bombyx mori. Insect Biochem. Mol. Biol. 2017, 81, 72-79. [CrossRef]

20. Zhang, W.; Ma, L.; Xiao, H.J.; Liu, C.; Chen, L.; Wu, S.L.; Liang, G.M. Identification and characterization of genes involving the early step of juvenile hormone pathway in helicoverpa armigera. Sci. Rep. 2017, 7, 1-12. [CrossRef]

21. Zhao, J.; Zhou, Y.; Li, X.; Cai, W.; Hua, H. Silencing of juvenile hormone epoxide hydrolase gene (Nljheh) enhances short wing formation in a macropterous strain of the brown planthopper, Nilaparvata lugens. J. Insect Physiol. 2017, 102, 18-26. [CrossRef]

22. Kalita, J. Heortia vitessoides Moore (Lepidoptera: Pyralidae)—A serious pest of Agarwood plant (Aquilaria malaccensis Lamk.). Geobios 2001, 29, 13-16.

23. Chen, J.X.; Lyu, Z.H.; Wang, C.Y.; Cheng, J.; Lin, T. RNA interference of a trehalose-6-phosphate synthase gene reveals its roles in the biosynthesis of chitin and lipids in Heortia vitessoides (Lepidoptera: Crambidae). Insect Sci. 2018. [CrossRef]

24. Livak, K.J.; Schmittgen, T.D. Analysis of relative gene expression data using real-time quantitative PCR and the $2^{-\Delta \Delta C T}$ Method. Methods 2001, 25, 402-408. [CrossRef]

25. Wei, D.; Liu, Y.W.; Zhang, Y.X.; Wang, J.J. Characterization and function of two short peptidoglycan recognition proteins involved in the immunity of Bactrocera dorsalis (Hendel). Insects 2019, 10, 79. [CrossRef]

26. Lu, Z.J.; Huang, Y.L.; Yu, H.Z.; Li, N.Y.; Xie, Y.X.; Zhang, Q.; Zeng, X.D.; Hu, H.; Huang, A.J.; Yi, L.; et al. Silencing of the chitin synthase gene is lethal to the Asian citrus psyllid, Diaphorina citri. Int. J. Mol. Sci. 2019, 20, 3734. [CrossRef]

27. Yoon, J.S.; Mogilicherla, K.; Gurusamy, D.; Chen, X.; Chereddy, S.C.R.R.; Palli, S.R. Double-stranded RNA binding protein, Staufen, is required for the initiation of RNAi in coleopteran insects. Proc. Natl. Acad. Sci. USA 2018, 115, 8334-8339. [CrossRef]

28. Xiong, K.C.; Wang, J.; Li, J.H.; Deng, Y.Q.; Pu, P.; Fan, H.; Liu, Y.L. RNA interference of a trehalose-6-phosphate synthase gene reveals its roles during larval-pupal metamorphosis in bactrocera minax (diptera: Tephritidae). J. Insect Physiol. 2016, 91, 84-92. [CrossRef] 
29. Ye, Y.X.; Pan, P.L.; Kang, D.; Lu, J.B.; Zhang, C.X. The multicopper oxidase gene family in the brown planthopper, Nilaparvata lugens. Insect Biochem. Mol. Biol. 2015, 63, 124-132. [CrossRef]

30. Wang, S.; Beerntsen, B.T. Functional implications of the peptidoglycan recognition proteins in the immunity of the yellow fever mosquito, Aedes aegypti. Insect Mol. Biol. 2015, 24, 293-310. [CrossRef]

31. Li, S.; Zhang, Q.R.; Xu, W.H.; Schooley, D.A. Juvenile hormone diol kinase, a calcium-binding protein with kinase activity, from the silkworm, Bombyx mori. Insect Biochem. Mol. Biol. 2005, 35, 1235-1248. [CrossRef]

32. Dubrovsky, E.B. Hormonal cross talk in insect development. Trends Endocrinol. Metab. 2005, 1, 6-11. [CrossRef]

33. Jones, G. Molecular mechanisms of action of juvenile hormone. Annu. Rev. Entomol. 1995, 40, 147-169. [CrossRef]

34. Parthasarathy, R.; Tan, A.; Sun, Z.; Chen, Z.; Rankin, M.; Palli, S.R. Juvenile hormone regulation of male accessory gland activity in the red flour beetle, Tribolium castaneum. Mech. Dev. 2009, 126, 563-579. [CrossRef]

35. Li, Z.L. Study on the Effects of Sublethal Concentration of Insecticides on Juvenile Hormone Metabolic Enzymes and Insulin-Like Genes of Nilaparvata Lugens Stal (Hemiptera: Delphacidae); Yangzhou University: Yangzhou, China, 2017.

36. Mirth, C.K.; Riddiford, L.M. Size assessment and growth control: How adult size is determined in insects. Bioessay 2007, 29, 344-355. [CrossRef]

37. Dhadialla, T.S.; Carlson, G.R.; Le, D.P. New insecticides with ecdysteroidal and juvenile hormone activity. Annu. Rev. Entomol. 1998, 43, 545-569. [CrossRef]

38. Jindra, M.; Palli, S.R.; Riddiford, L.M. The juvenile hormone signaling pathway in insect development. Annu. Rev. Entomol. 2013, 58, 181-204. [CrossRef]

39. Arrese, E.L.; Soulages, J.L. Insect fat body: Energy, metabolism, and regulation. Annu. Rev. Entomol. 2010, 55, 207-225. [CrossRef]

40. Sun, B.B. Effects of Juvenile Hormone and Low Temperature on Flight and Reproduction of the Rice Leaf Roller, Cnaphalocrocis medinalis; Chinese Academy of Agricultural Sciences: Beijing, China, 2012.

(C) 2019 by the authors. Licensee MDPI, Basel, Switzerland. This article is an open access article distributed under the terms and conditions of the Creative Commons Attribution (CC BY) license (http://creativecommons.org/licenses/by/4.0/). 